\title{
Molecular characterization of class 1, 2 and 3 integrons in clinical multi-drug resistant Klebsiella pneumoniae isolates
}

\author{
Farzaneh Firoozeh ${ }^{1,2}$, Zeinab Mahluji ${ }^{2}$, Ahmad Khorshidi $^{2}$ and Mohammad Zibaei ${ }^{3,4^{*}}$ (D)
}

\begin{abstract}
Background: The aim of this study was to characterize class 1,2 and 3 integrons in clinical MDR Klebsiella pneumoniae isolates in Kashan, Iran.

Methods: One hundred-eighty one Klebsiella pneumoniae were recovered from clinical specimens during November 2013 to October 2014. Antimicrobial susceptibility patterns were determined by disk diffusion method according to the Clinical and Laboratory Standards Institute (CLSI) guidelines for detection of MDR strains. Of the 181 Klebsiella pneumoniae, 146 (80.7\%) of isolates were isolated from nosocomial infected patients and 150 (82.9\%) identified as MDR isolates. The PCR amplification was used to show presence of class 1, 2 and 3 integrons among MDR strains. The PCR method and sequencing were used for evaluation of cassette content of integrons.

Results: Of the MDR K. pneumoniae isolates, 150 (100\%) and 55 (36.7\%) carried int/1 and int/2 genes, respectively. None of the MDR Klebsiella pneumoniae isolates carried class 3 integrons. Amplification of conserved segment (CS) of class 1 and class 2 integrons revealed 10 different arrays including: No. cassette; dfrA5, dfrA30; aadA2; aadA2, dfrA12; dfrA17, aadA5, aadA4; dfrA5, dfrA30, aadA2; dfrA5, dfrA30, aadA2, dfrA12, dfrA5, dfrA30, dfrA17, aadA5, aadA4; aadA2, aadA2, dfrA12; dfrA5, dfrA30, aadA2, aadA2, dfrA12 and 4 arrays including: No. cassette; aadA1; dfrA1-sat1; aadA1, dfrA1-sat1, respectively.
\end{abstract}

Conclusions: The finding of present study revealed a high prevalence of integrons especially class 1 among MDR $K$. pneumoniae isolates from nosocomial infections in Kashan, which led to rapid extension of MDR strains.

Keywords: Klebsiella pneumoniae, Multidrug resistance, Integrons class 1, Integrons class 2, Integrons class 3, Cassette contents

\section{Background}

Klebsiella pneumoniae (K. pneumoniae) is one of the most important causes of infection especially among hospitalized patients [1]. Multi-drug resistant (MDR) $K$. pneumoniae isolates are becoming increasingly prevalent in the clinical and nosocomial environments and is raised as a major threat in treatment of nosocomial infections [2]. Different mechanisms and factors involved in the development and spread of antibiotic resistance in bacterial strains. Among them acquisition of resistance

\footnotetext{
* Correspondence: zibaeim@sums.ac.ir

${ }^{3}$ Evidence-based Phytotherapy \& Complementary Medicine Research Center,

Alborz University of Medical Sciences, Karaj, Iran

${ }^{4}$ Department of Parasitology and Mycology, School of Medicine, Alborz

University of Medical Sciences, P.O. Box: 3149779453, Karaj, Iran

Full list of author information is available at the end of the article
}

genes especially via mobile genetic elements is considered as the main factor in the wide distribution of antimicrobial resistance [3]. Integrons which are one of the kind mobile genetic elements presumed to be involved in the dissemination of these MDR strains [4]. Integrons are considered powerful mobile genetic elements that are located on plasmids, transposons and pathogenicity islands which facilitate their transferring among different bacteria. According to reports available, integrons have a wide distribution among clinically isolated bacteria; also, their mobility has become a major problem in antibiotic resistance in clinical specimens [5]. Till now, five classes of integrons have been described based on the nucleotide sequence of the integrase gene [6].

(c) The Author(s). 2019 Open Access This article is distributed under the terms of the Creative Commons Attribution 4.0 International License (http://creativecommons.org/licenses/by/4.0/), which permits unrestricted use, distribution, and reproduction in any medium, provided you give appropriate credit to the original author(s) and the source, provide a link to the Creative Commons license, and indicate if changes were made. The Creative Commons Public Domain Dedication waiver (http://creativecommons.org/publicdomain/zero/1.0/) applies to the data made available in this article, unless otherwise stated. 
Class 1 integrons are the most prevalent and have been frequently reported in clinical isolates of gram negative bacteria including $K$. pneumoniae [7]. The structure of class 1 integrons is consisted of two conserved regions, including $3^{\prime}$ conserved segment ( $3^{\prime} \mathrm{CS}$ ) and $5^{\prime}$ conserved segment $\left(5^{\prime} \mathrm{CS}\right)$, as well as internal gene cassettes that encode antimicrobial resistance genes [7]. Class 2 integrons found sometimes and class 3 integrons are rarely documented in $K$. pneumoniae [3]. Up to now, more than 130 different cassettes which confer resistance against a wide range of antibiotics including all ß- lactams, all aminoglycosides, quinolones, fluoroquinolones, macrolides, and many other antibiotics classes have been detected [5]. Integrons carrying diverse cassette arrays have been identified in different studies in Europe and Asia [5]. Class 1 integrons carries variety of resistance gene cassettes, and most of them contain $\operatorname{aad} A$ gene which confer resistance to streptomycinspectinomycin. The wide spread distribution of class 1 integrons harbouring different alleles of the aadA gene has been documented [5]. Also, the $d f r A$ cassette arrays, encoding resistance to trimethoprim, are frequently detected in both class 1, and 2 integrons [8]. Although several studies have documented the prevalence of integrons in MDR K. pneumoniae isolated from clinical specimens in Iran $[9,10]$, there is little information regarding the association between class 2 and 3 integrons and MDR, also cassettes contents of integrons in K. pneumoniae isolates from nosocomial infected patients in our region. Thus, the present study proposed to characterize class 1, 2 and 3 integrons in clinical MDR K. pneumoniae isolates in Kashan, Iran.

\section{Methods}

\section{Bacterial isolates}

A total of 181 non-duplicate $K$. pneumoniae isolates from clinical specimens at Shahid Beheshti Hospital in Kashan, Iran, during November 2013 to October 2014 were enrolled in the study. All patients admitted to Shahid Beheshti Hospital in Kashan and diagnosed with infections caused by $K$. pneumoniae during the study were included. While, patients diagnosed with infections due to other bacteria than $K$. pneumoniae during the same time, were excluded. Of the $181 \mathrm{~K}$. pneumoniae isolates, $146(80.7 \%)$ were nosocomial and had been occurred after $48 \mathrm{~h}$ of hospital admission. The isolates were from both sexes including 78 male and 103 female and were recovered from urine, respiratory tract samples (sputum, bronchoalveolar lavage, tracheal aspirate and nasal discharge), blood, wound, cerebrospinal fluid (CSF) and catheter. Collection of specimens from the lower respiratory tract was done by pulmonologists prior to initiation of antimicrobial therapy. Tracheal aspirate obtained from patients by instillation of $1.5 \mathrm{~mL}$ saline $(\mathrm{pH}$
7.0) in to each nostril. After insertion of plastic catheter or tubing contained $2 \mathrm{~mL}$ of saline into the nostril, nasopharyngeal secretions were aspirated and collected in sterile containers. Bronchoalveolar lavage (BAL) was obtained using bronchoscope. Also deep cough sputum of other patients with respiratory infections were collected in sterile containers. The collection of CSF was performed by clinicians under aseptic conditions. In addition, in order to obtain urine specimen from patients with urinary tract infection (UTI) and indwelling catheter, sterile syringe was inserted in to catheter at a 45 degree angle, then $20-30 \mathrm{~mL}$ of urine was withdrawn and collected in sterile containers.

The isolates were identified as $K$. pneumoniae, after culturing on MacConkey agar (Merck, Germany) media and incubation at $37^{\circ} \mathrm{C}$ for $24 \mathrm{~h}$. Characteristic colonies of $K$. pneumoniae were confirmed by standard biochemical tests including TSI (Triple Sugar Iron Agar), Indole, Methyl Red (MR), Voges-Proskauer (VP), and Citrate (IMVIC), also urease and motility tests (Merck, Germany). All K. pneumoniae isolates were confirmed as, Indole negative, MR negative, VP positive, Citrate positive, urease positive, and motility negative gram negative coccobacilli [11].

\section{Antibiotic susceptibility testing}

K. pneumoniae isolates were tested for susceptibility to antimicrobial agents and identification of MDR strains were done by using disk diffusion method described by the Clinical and Laboratory Standards Institute (CLSI) guidelines [12]. The antibiotics were selected according to CLSI standard and previous studies in this field as follows: ampicillin $(30 \mu \mathrm{g})$, amoxicillin/clavulanic acid $(20 / 10 \mu \mathrm{g})$, aztreonam $(30 \mu \mathrm{g})$, cephalothin $(30 \mu \mathrm{g})$, cefotaxime $(30 \mu \mathrm{g})$, ceftazidime $(30 \mu \mathrm{g})$, cefoxitin $(30 \mu \mathrm{g})$, cefteriaxon $(30 \mu \mathrm{g})$, imipenem $(10 \mu \mathrm{g})$, gentamicin $(10 \mu \mathrm{g})$, nalidixic acid $(30 \mu \mathrm{g})$ and ciprofloxacin $(5 \mu \mathrm{g})$ (Mast Companies, UK). The E. coli strain ATCC 25922 was used as a control. Results were interpreted according CLSI and the manufacturer protocols (Mast, UK) and each $K$. pneumoniae isolate which showed resistance to more than three antibiotic classes was identified as MDR [13].

\section{Genomic DNA extraction}

DNA of each MDR K. pneumoniae isolates was extracted by boiling method. The overnight cultures of K. pneumoniae strains in LB broth were suspended in $250 \mu \mathrm{L}$ of sterile deionized water and incubated at $100{ }^{\circ} \mathrm{C}$ for $10 \mathrm{~min}$. After centrifugation at $10,000 \mathrm{~g}$ for $5 \mathrm{~min}$, the supernatant were used as a template DNA and stored at $-20^{\circ} \mathrm{C}$ until use [14]. 


\section{Detection and characterization of class 1, 2 and 3 integrons}

The presence of class 1, 2 and 3 integrons in MDR $K$. pneumoniae were investigated by amplification of integrase genes including intI1, intI2, and intI3 specific primers (Table 1). The PCR reactions were prepared in a total volume of $25 \mu \mathrm{L}$ and amplification was performed in a thermaocycler (Eppendorf master cycler ${ }^{\circledR}, \mathrm{MA}$ ) as follows: $5 \mathrm{~min}$ at $94{ }^{\circ} \mathrm{C} ; 35$ cycles of $1 \mathrm{~min}$ at $94{ }^{\circ} \mathrm{C}, 1 \mathrm{~min}$ at $55^{\circ} \mathrm{C}, 30 \mathrm{~s}$ at $72{ }^{\circ} \mathrm{C} ; 10 \mathrm{~min}$ at $72{ }^{\circ} \mathrm{C}$ for detection of intI1 gene and $5 \mathrm{~min}$ at $94^{\circ} \mathrm{C} ; 32$ cycles of $1 \mathrm{~min}$ at $94{ }^{\circ} \mathrm{C}, 1 \mathrm{~min}$ at $60{ }^{\circ} \mathrm{C}, 2 \mathrm{~min}$ at $72{ }^{\circ} \mathrm{C} ; 10 \mathrm{~min}$ at $72{ }^{\circ} \mathrm{C}$ for detection of intI2, and intI3 genes [15-17]. Reaction mixtures without a DNA template used as negative control. The amplified products were electrophoresed on $1.2 \%$ agarose gel and after staining with ethidium bromide $(0.5 \mathrm{mg} / \mathrm{ml})$ visualized in gel document system (Biorad, UK).

\section{Detection and characterization of integrons internal variable region genes}

All integron-positive MDR $K$. pneumoniae isolates were tested for the presence of internal cassettes genes by CS-PCR using 3'CS and 5'CS primers (Table 1). The following conditions was used for CS-PCR reaction: initial denaturation at $94{ }^{\circ} \mathrm{C}$ for $5 \mathrm{~min}$, followed by 35 cycles of denaturation at $94{ }^{\circ} \mathrm{C}$ for $1 \mathrm{~min}$, annealing at $58{ }^{\circ} \mathrm{C}$ for $1 \mathrm{~min}$, and extension at $72^{\circ} \mathrm{C}$ for $2 \mathrm{~min}$, with final extension at $72{ }^{\circ} \mathrm{C}$ for $10 \mathrm{~min}$ [18]. After electrophoresis on $1.2 \%$ agarose gel and staining, the PCR products were visualized.

\section{DNA sequencing and integrons gene cassettes analysis}

The purified PCR products were sequenced by the ABI Capillary System and Sanger's method (Macrogen Research, Seoul, Korea) using $10 \mathrm{pmol}$ of specific primers. The sequences were analyzed by Chromas Pro version1.7.5 Technelysium as well as online BLAST software (http://www.ncbi.nlm. nih.gov/BLAST/). Sequences of CS-PCR products revealed the integrons cassettes contents.

\section{Results}

This study was conducted on 181 patients with age ranged between 1 and 97 (mean $50.36 \pm 3.80$ years). The K. pneumoniae were isolated from clinical specimens including: urine $124(68.5 \%)$, wound $6(3.3 \%)$, blood 5 (2.8\%), respiratory tract samples $43(23.8 \%)$ including $(6$ sputum, 2 bronchoalveolar lavage, 34 tracheal aspirates and 1 nasal discharge), CSF $1(0.6 \%)$ and catheter 2 (1.1\%).

The antibiotic susceptibility patterns by disk diffusion are shown in (Fig. 1). The highest resistance was obtained to ampicillin, cephalothin, cefotaxime and cefteriaxon. One hundred- fifty (82.9\%) identified as MDR isolates, and showed resistance to more than three antimicrobial families. Class 1 and 2 integrons were detected in $150(100 \%)$ and 55(36.7\%) of MDR K. pneumoniae isolates, which showed to carry intI1 and intI2 genes respectively. The intI3 gene was not identified among 150 MDR K. pneumoniae isolates and class 3 integrons were not founded in any MDR K. pneumoniae isolates.

Sequencing analysis for intI-positive strains revealed that the cassette arrays of class 1 integron were including 10 different arrays groups from A-J (Table 2), consist of (708 bp, $1002 \mathrm{bp}, 1500 \mathrm{bp}$ and $1610 \mathrm{bp}$ integrons) and identified gene cassettes were as follows: (no cassette; dfrA5, dfrA30; aadA2; aadA2, dfrA12; dfrA17, aadA5, $\operatorname{aadA4} ; d f r A 5, d f r A 30, \operatorname{aad} A 2 ; d f r A 5, d f r A 30, \operatorname{aad} A 2$, dfrA12; dfrA5, dfrA30, dfrA17, aadA5, aadA4; aadA2, aadA2, dfrA12; dfrA5, dfrA30, aadA2, aadA2, dfrA12) Whereas, 4 different cassette arrays groups from a-d (Table 3) consist of (1000 bp and $1500 \mathrm{bp}$ integrons) were detected among 55 MDR $K$. pneumoniae isolates which carried class 2 integrons, and identified gene cassettes were as follows: (no cassette; aadA1; dfrA1-sat1; $\operatorname{aadA1,dfrA1-sat1).}$

Table 1 Primers used for PCR amplification for detection of class 1, 2 and 3 integrons

\begin{tabular}{|c|c|c|c|c|}
\hline Gene & Primer & Sequence $\left(5^{\prime}-3^{\prime}\right)$ & Amplification Product (bp) & Reference \\
\hline \multirow[t]{2}{*}{ int/1 } & Intl1-F & TCTCGGGTAACATCAAGG & 254 & {$[11]$} \\
\hline & Intl-R & AGGAGATCCGAAGACCTC & & \\
\hline \multirow[t]{2}{*}{ int 12} & $\ln t \mid 2-\mathrm{F}$ & CACGGATATGCGACAAAAAGG & 788 & [12] \\
\hline & $\ln t \mid 2-R$ & TGTAGCAAACGAGTGACGAAATG & & \\
\hline \multirow[t]{2}{*}{ int/3 } & Intl3-F & AGTGGGTGGCGAATGAGTG & 600 & {$[13]$} \\
\hline & Intl3-R & TGTTCTTGTATCGGCAGGTG & & \\
\hline $5^{\prime} \mathrm{CS}$ & $5^{\prime} \mathrm{CS}-\mathrm{F}$ & GGCATCCAAGCAGCAAG & Variable & {$[14]$} \\
\hline $3^{\prime} \mathrm{CS}$ & $3^{\prime} \mathrm{CS}-\mathrm{R}$ & AAGCAGACTTGACCTGA & & \\
\hline$a t t / 2$ & att|2-F & GACGGCATGCACGATTTGTA & Variable & [14] \\
\hline orf $x$ & orfX-R & GATGCCATCGCAAGTACGAG & & \\
\hline
\end{tabular}




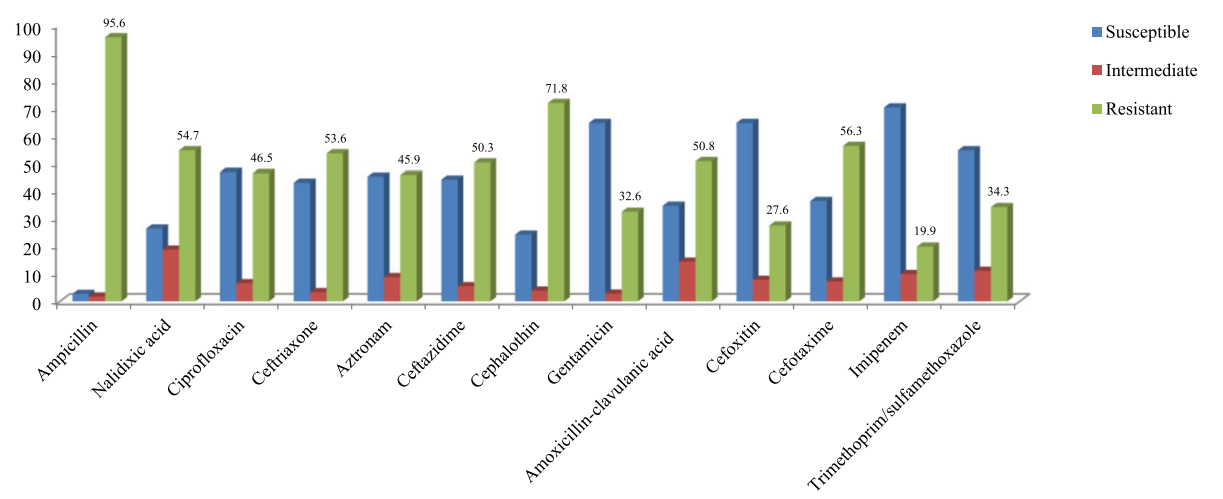

Fig. 1 The antibiotic resistant patterns of $K$. pneumoniae isolates measured by disk diffusion method $(N=181)$

Table 2 Sources, numbers, sizes, inserted cassettes and groups of cassettes arrays of integrons, among integron class 1-positive K. pneumoniae isolates

\begin{tabular}{|c|c|c|c|c|c|c|c|}
\hline Source & $\begin{array}{l}\text { Isolate }(\mathrm{N}) \\
(\%)\end{array}$ & $\begin{array}{l}\text { int/1 } \\
(\mathrm{No}=150)\end{array}$ & $\begin{array}{l}\mathrm{CS}_{1} \\
(\mathrm{No}=132)\end{array}$ & $\begin{array}{l}\text { NO. of Integrons } \\
\text { Class1 }\end{array}$ & $\begin{array}{l}\text { Integron } \\
\text { Sizes(s) (bp) }\end{array}$ & Inserted Cassette(s) & $\begin{array}{l}\text { Groups of Cassettes } \\
\text { Arrays of Integrons } \\
\text { Class } 1\end{array}$ \\
\hline \multirow[t]{10}{*}{ Urine } & $14(9.3)$ & + & - & - & - & NO cassette & A \\
\hline & 25 (16.6) & + & + & 2 & 708 & dfrA5, dfrA30 & B \\
\hline & $3(2.0)$ & + & + & 1 & 1002 & $\operatorname{aadA2}$ & C \\
\hline & $13(8.7)$ & + & + & 2 & 1500 & $\operatorname{aad} A 2, \operatorname{dfr} A 12$ & $\mathrm{D}$ \\
\hline & $10(6.7)$ & + & + & 3 & 1610 & dfrA17, aadA5, aadA4 & $E$ \\
\hline & $7(4.6)$ & + & + & 3 & 708,1002 & dfrA5, dfrA30, aadA2 & $\mathrm{F}$ \\
\hline & $13(8.7)$ & + & + & 4 & 708,1500 & dfrA5, dfrA30, aadA2, dfrA12 & G \\
\hline & $2(1.3)$ & + & + & 5 & 708,1610 & dfrA5, dfrA30, dfrA17, aadA5, aadA4 & $\mathrm{H}$ \\
\hline & $4(2.6)$ & + & + & 3 & 1002,1500 & $\operatorname{aad} A 2, \operatorname{aad} A 2, \mathrm{dfr} A 12$ & 1 \\
\hline & $8(5.3)$ & + & + & 5 & $708,1002,1500$ & dfrA5, dfrA30, aadA2, aadA2, dfrA12 & J \\
\hline \multirow[t]{9}{*}{ Respiratory } & $3(2.0)$ & + & + & & - & NO cassette & A \\
\hline & $15(10)$ & + & + & 2 & 708 & dfrA5, dfrA30 & B \\
\hline & $1(0.7)$ & + & + & 1 & 1002 & $\operatorname{aadA2}$ & C \\
\hline & $10(6.7)$ & + & + & 2 & 1500 & $\operatorname{aad} A 2, \mathrm{dfr} A 12$ & D \\
\hline & $6(4.0)$ & + & + & 3 & 1610 & dfrA17, aadA5, aadA4 & $E$ \\
\hline & $2(1.3)$ & + & + & 3 & 708,1002 & dfrA5, dfrA30, aadA2 & $\mathrm{F}$ \\
\hline & $1(0.7)$ & + & + & 4 & 708,1500 & dfrA5, dfrA30, aadA2, dfrA12 & G \\
\hline & $1(0.7)$ & + & + & 3 & 1002,1500 & $\operatorname{aad} A 2, \operatorname{aad} A 2, \mathrm{dfr} A 12$ & 1 \\
\hline & $1(0.7)$ & + & + & 5 & $708,1002,1500$ & dfrA5, dfrA30, aadA2, aadA2, dfrA12 & J \\
\hline \multirow[t]{2}{*}{ Blood } & $1(0.7)$ & + & + & 3 & 1610 & dfrA17, aadA5, aadA4 & $E$ \\
\hline & $1(0.7)$ & + & + & 3 & 708,1002 & dfrA5, dfrA30, aadA2 & $\mathrm{F}$ \\
\hline \multirow[t]{2}{*}{ Wound } & $3(2.0)$ & + & + & 2 & 708 & dfrA5, dfrA30 & B \\
\hline & $3(2.0)$ & + & + & 3 & 1610 & dfrA17, aadA5, aadA4 & $\mathrm{E}$ \\
\hline CSF & $1(0.7)$ & + & + & & - & NO cassette & A \\
\hline Catheter & $2(1.3)$ & + & + & 2 & 1500 & $\operatorname{aad} A 2, \operatorname{dfr} A 12$ & $\mathrm{D}$ \\
\hline
\end{tabular}


Table 3 Sources, numbers, sizes, inserted cassettes and groups of cassettes arrays of integrons, among integron class 2-positive K. pneumoniae isolates

\begin{tabular}{|c|c|c|c|c|c|c|c|}
\hline Source & Isolate N (\%) & int/2 ( $\mathrm{No}=55)$ & $\mathrm{Cs}_{2}(\mathrm{No}=37)$ & $\begin{array}{l}\text { NO. of Integrons } \\
\text { Class } 2\end{array}$ & Integron Sizes(s) (bp) & Inserted Cassette(s) & $\begin{array}{l}\text { Groups of Cassettes } \\
\text { Arrays of Integrons Class } 2\end{array}$ \\
\hline \multirow[t]{4}{*}{ Urine } & $12(21.9)$ & + & - & - & - & NO cassette & a \\
\hline & $7(12.7)$ & + & + & 1 & 1000 & $\operatorname{aadA1}$ & $b$ \\
\hline & $6(10.9)$ & + & + & 2 & 1500 & dfrA1-sat1 & c \\
\hline & $7(12.7)$ & + & + & 3 & 1000,1500 & aadA1, dfrA1-sat1 & $d$ \\
\hline \multirow[t]{4}{*}{ Respiratory } & $3(5.5)$ & + & - & - & - & NO cassette & a \\
\hline & $3(5.5)$ & + & + & 1 & 1000 & $\operatorname{aadA1}$ & $b$ \\
\hline & $6(10.9)$ & + & + & 2 & 1500 & dfra1-sat1 & c \\
\hline & $4(7.3)$ & + & + & 3 & 1000,1500 & aadA1, dfrA1-sat1 & $d$ \\
\hline \multirow[t]{2}{*}{ Blood } & $1(1.8)$ & + & - & - & - & NO cassette & $a$ \\
\hline & $1(1.8)$ & + & + & 3 & 1000,1500 & aadA1, dfrA1-sat1 & $d$ \\
\hline \multirow[t]{3}{*}{ Wound } & $2(3.6)$ & + & - & - & - & NO cassette & $\mathrm{a}$ \\
\hline & $1(1.8)$ & + & + & 1 & 1000 & $\operatorname{aadA1}$ & $b$ \\
\hline & $2(3.6)$ & + & + & 3 & 1000,1500 & aadA1, dfrA1-sat1 & $d$ \\
\hline
\end{tabular}

The most common cassettes were $708 \mathrm{bp}$, which were detected in $43(28.6 \%)$ isolates with class 1 integrons (Table 2), whereas among class 2 integrons, the most frequent cassettes were 1000-1500 bp, which were identified among $14(25.5 \%)$ of them (Table 3). Twentythree $(15.3 \%)$ of class 1 integron positive $K$. pneumoniae strains carried more than three gene cassettes simultaneously consisting of array of 708,1500 bp; 708, $1610 \mathrm{bp}$; 708, 1002, $1500 \mathrm{bp}$.

\section{Discussion}

MDR K. pneumoniae has become an important challenge in treatment of nosocomial infections worldwide [19]. It has been documented that mobile genetic elements, such as integrons, play an important role in the dissemination of MDR- K. pneumoniae isolates [20]. In this study we have characterized the class 1,2 and 3 integrons in clinical MDR $K$. pneumoniae isolates in Iran. All MDR K. pneumoniae that we investigated were positive for class 1 integrons. The intense association between the presence of class 1 integrons and occurrence of MDR among gram negative bacteria has been documented [21, 22]. In $\mathrm{Li}$ et al. [21] study, the class 1 integron positive isolates in comparison with class 1 integron negative isolates showed resistance to a much higher number of drugs [21]. High frequency of integron positive MDR- K. pneumoniae has been reported from other studies [22, 23]. High Prevalence of integrons among MDR strains, could be due to that integrons confer a selection advantage to strains that live in environments such a hospitals where selective pressures created by overuse of antibiotics. We found that among the integron class 1 positive strains, 10 amplicon were identified. The most prevalent arrays observed among class 1 integrons were $708 \mathrm{bp}$ and gene cassettes identified were $d f r A 5$ and $d f r A 30$ which encode dihydrofolate reductases enzymes. Also other variants of $d f r A$ genes including $d f r A 12$ and $d f r A 17$ were identified in relatively high frequency among our integron class 1 positive $K$. pneumonia strains. The studies show that the most prevalent integron cassette- associated genes are those encode dihydrofolate reductases and aminoglycoside modifying enzymes [21, 24-26]. Salimizand et al. [9] reported dfrA17 variant in Klebsiella species. The cassette arrays of class 1 integron in intI1-positive $K$. pneumoniae strains in China were included $d f r A 17, d f r A 12, d f r A 1$, $d$ fr $A 25, d f r A 27$, genes $[19,21]$. The $d f r A 17$ and $d f r A 12$ have been identified among gram negative bacteria that carried class 1 integrons in USA [27], which shows these variants are common among cassettes of class 1 integrons in the world. In other studies in Iran other variants of $d f r A$ genes including $d f r A 7, d f r A 1 d f r A 25$, $d f r A 5$ and $d f r A 12$ have been documented among Salmonella serotypes and Enteropathogenic Escherichia coli (EPEC) isolates respectively [24, 28-30]. To our knowledge this is the first report of detecting $d f r A 5, d f r A 12$ and $d$ frA30 variants among $K$. pneumoniae in Iran. Also detection of $d f r A 5, d f r A 12$ variants in other species could be due to interspecies gene transfer. The second most prevalent cassette in our study was aadA2 which were identified in 67 intI1-positive $K$. pneumoniae strains. The frequency of presence of other variants of $\operatorname{aad} A$ genes including $\operatorname{aad} A 4$ and $\operatorname{aad} A 5$ were also relatively high in studied intI1-positive $K$. pneumoniae strains. Till now, 18 different variants of the $\operatorname{add} A$ genes which encode resistance to streptomycin- spectinomycin have been identified on gene cassettes of class 1 integrons among gram-negative bacteria [26]. The presence 
of aadA2 is shown in Salmonella serotypes and EPEC with low prevalence in Iran $[24,30]$. In studies conducted in Taiwan, aadA genes variants including aadA1, $\operatorname{aad} A 2$ and aadA4 have been reported in majority of MDR Acinetobacter baumannii isolates and integrons class 1 carrying aadA2 variant was the most frequently found cassette array in clinical isolates of $K$. pneumoniae $[31,32]$. In a study in Iran aadA5 gene have been identified among integron cassettes of MDR Klebsiella spp. [9]. According to data in the literature this is the first report of aadA2 variant in K. pneumoniae in Iran and presence of this gene in different studies among cassettes arrays of class 1 integrons shows that this gene may be the first cassette to be captured by an integron. Another interesting founding was that relatively high frequency of class 1 integron positive $K$. pneumoniae strains carried more than three gene cassettes simultaneously. This result in correlation with other studies in different geographical areas shows the high diversity of integrons among $K$. pneumoniae isolates in our regions [7, 32, 33]. The frequency of class 2 integrons in our MDR K. pneumoniae isolates was $36.7 \%$ which is higher than that reported by Ahangarzadeh Rezaee et al. [10] in northwest of Iran. The frequencies of 14, 4.8, and 10.4\% have been reported in Acinetobacter baumannii, EPEC and Escherichia coli isolates from humans and animals in Iran and different parts of the world respectively [34-37]. In study of cassettes contents of class 2 integrons in MDR K. pneumoniae isolates for the first time in Iran, we found that the most prevalent cassettes arrays observed among class 2 integrons were aadA1 and dfrA1-sat1 which confer resistance to streptomycin and spectinomycin, trimethoprim and streptothricin respectively. In other studied these cassettes arrays are frequently detected among class 2 integrons [18, 38]. In a study conducted by Eftekhari et al. [39], these cassettes were identified in class 2 integrons in Shigella spp. isolated from patients in Iran.

The high frequency of aadA1 and dfrA1-sat1 genes which has been documented in different reports, shows the stability of these gene cassettes among class 2 integrons especially in gram negative bacteria, also the identification of these gene cassettes among other genus of Enterobacteriaceae family is evidence of interspecies transition of class 2 integrons. We found an apparent association between the presence of aad and $d r f$ gene cassette arrays and phenotypic resistance to the corresponding antibiotics in our int-positive strains. On the other hand, despite the observation of high phenotypic resistance to many other antibiotics such as ampicillin, no cassette carrying the gene for resistance to these antibiotics was obtained. This could be due to the fact that the resistance genes to these antibiotics are located outside the integrons. Of the all integron class 1 and 2 positive K. pneumoniae strains, 12.0 and $37.2 \%$ isolates harboured empty integrons and did not carried any gene cassettes. Empty integrons have been documented by other reports $[18,39]$ indicate the potential of these isolates to capture resistance gene cassettes and change to strains with multiple resistance determinants especially in hospitals environments due to antibiotic selective pressures.

\section{Conclusion}

Over all, the results of in this study revealed a high prevalence of integrons especially class 1 among MDR $K$. pneumoniae isolates from nosocomial infections in Kashan, which led to rapid extension of MDR strains.

\section{Acknowledgements \\ The technical assistance of the staff of Microbiology Laboratory, of the Department of Microbiology and Immunology, School of Medicine, Kashan University of Medical Sciences are gratefully acknowledged. The results described in this paper formed part of an MSc student thesis.}

\section{Funding}

Nil.

\begin{abstract}
Availability of data and materials
The data analyzed during the study will be available from the corresponding authors upon request.
\end{abstract}

\section{Authors' contributions}

FF designed the study, collected and analyzed the data, drafted the paper, and supervised the overall work. ZM contributed on the data collection and statistical analysis. AK reviewed the study design. MZ contributed on the interpretation of the data, the study design of the paper, and approved the manuscript. All authors have accepted and approved the final version of the manuscript.

\section{Ethics approval and consent to participate}

The purpose and procedures of the study were explained to all participants, and a written informed consent was obtained from all of them. The study was approved by the ethical clearance committees of the Kashan University of Medical Sciences.

\section{Consent for publication}

Not applicable.

\section{Competing interests}

The authors declare that they have no competing interests.

\section{Publisher's Note}

Springer Nature remains neutral with regard to jurisdictional claims in published maps and institutional affiliations.

\section{Author details}

${ }^{1}$ Department of Microbiology, School of Medicine, Alborz University of Medical Sciences, Karaj, Iran. ${ }^{2}$ Department of Microbiology, School of Medicine, Kashan University of Medical Sciences, Kashan, Iran.

${ }^{3}$ Evidence-based Phytotherapy \& Complementary Medicine Research Center, Alborz University of Medical Sciences, Karaj, Iran. ${ }^{4}$ Department of Parasitology and Mycology, School of Medicine, Alborz University of Medical Sciences, P.O. Box: 3149779453, Karaj, Iran. 


\section{Received: 27 November 2018 Accepted: 15 March 2019} Published online: 29 March 2019

\section{References}

1. Tsai YK, Fung CP, Lin JC, Chen JH, Chang FY, Chen TL, et al. Klebsiella pneumonia outer membrane porins OmpK35 and OmpK36 play roles in both antimicrobial resistance and virulence. Antimicrob Agents Chemother. 2011;55:1485-93.

2. Lombardi F, Gaia P, Valaperta R, Cornetta M, Tejada MR, Girolamo LD, et al. Emergence of carbapenem-resistant Klebsiella pneumoniae: progressive spread and four-year period of observation in a cardiac surgery division. Biomed Res Int. 2015;2015:871947.

3. Odumosu BT, Adeniyi BA, Chandra R. Analysis of integrons and associated gene cassettes in clinical isolates of multidrug resistant Pseudomonas aeruginosa from Southwest Nigeria. Ann Clin Microbiol Antimicrob. 2013;12:29.

4. Essen-Zandbergen A, Smith H, Veldman K, Mevius D. Occurrence and characteristics of class 1, 2 and 3 integrons in Escherichia coli, Salmonella and Campylobacter spp. in the Netherlands. J Antimicrob Chemother. 2007; 59:746-50.

5. Deng Y, Bao X, Ji L, Chen L, Liu J, Miao J, et al. Resistance integrons: class 1, 2 and 3 integrons. Ann Clin Microbiol Antimicrob. 2015;14:45.

6. Guerin E, Jove T, Tabesse A, Mazel D, Ploy MC. High-level gene cassette transcription prevents integrase expression in class 1 integrons. J Bacteriol. 2011:193:5675-82

7. Lima AM, de Melo ME, Alves LC, Brayner FA, Lopes AC. Investigation of class 1 integrons in Klebsiella pneumoniae clinical and microbiota isolates belonging to different phylogenetic groups in Recife, State of Pernambuco. Rev Soc Bras Med Trop. 2014;47:165-9.

8. Kiiru J, Butaye P, Goddeeris BM, Kariuki S. Analysis for prevalence and physical linkages amongst integrons, ISEcP1, ISCR1, Tn21 and Tn7 encountered in Escherichia coli strains from hospitalized and nonhospitalized patients in Kenya during a 19-year period (1992-2011). BMC Microbiol. 2013;13:109.

9. Salimizand H, Shahcheraghi F, Kalantar E, Badmasti F, Mousavi SF. Molecular characterization of class 1 integrons and gene cassettes in multidrug resistant (MDR) Klebsiella spp. isolated from hospitalized and outpatients in Iran, 2009. Iran J Microbiol. 2013:5:48-55.

10. Ahangarzadeh-Rezaee M, Langarizadeh N, Aghazadeh M. First report of class 1 and class 2 integrons in multidrug-resistant Klebsiella pneumoniae isolates from Northwest Iran. Jpn J Infect Dis. 2012;65:256-9.

11. Mahon CR, Lehman DC, Manuselis G. Textbook of diagnostic microbiology. USA: Saunders Company; 2011. p. 427-61.

12. Clinical and Laboratory Standards Institute: Performance Standards for Antimicrobial Susceptibility Testing. Wayne PA, USA 2013. 23th information supplement, M100-S23.

13. Amiri A, Firoozeh F, Moniri R, Zibaei M. Prevalence of CTX-M-type and PER extended-spectrum $\beta$-lactamases among Klebsiella spp. isolated from clinical specimens in the teaching hospital of Kashan, Iran. Iran Red Crescent Med J. 2016;18:e22260

14. Afzali H, Firoozeh F, Amiri A, Moniri R, Zibaei M. Characterization of CTX-Mtype extend-spectrum $\beta$-lactamase producing Klebsiella spp. in Kashan, Iran. Jundishapur J Microbiol. 2015;8:e27967.

15. Leverstein-van Hall MA, Blok HEM, Donders ART, Paauw A, Fluit AC, Verhoef J. Multidrug resistance among Enterobacteriaceae is strongly associated with the presence of integrons and is independent of species or isolate origin. J Infect Dis. 2003;187:251-9.

16. Machado E, Ferreira J, Novais A, Peixe L, Canton R, Baquero F, et al. Preservation of integron types among Enterobacteriaceae producing extended-spectrum $\beta$-lactamases in a Spanish hospital over a 15-year period (1988 to2003). Antimicrob Agents Chemother. 2007;51:2201-4.

17. Goldstein C, Lee MD, Sanchez S, Hudson C, Phillips B, Register B, et al. Incidence of class 1 and 2 integrases in clinical and commensal bacteria from livestock, companion animals, and exotics. Antimicrob Agents Chemother. 2001:45:723-6.

18. Lapierre L, Cornejo J, Borie C, Toro C, San Martín B. Genetic characterization of antibiotic resistance genes linked to class 1 and class 2 integrons in commensal strains of Escherichia coli isolated from poultry and swine. Microb Drug Resist. 2008;14:265-72

19. Cao X, Xu X, Zhang Z, Shen H, Chen J, Zhang K. Molecular characterization of clinical multidrug-resistant Klebsiella pneumoniae isolates. Ann Clin Microbiol Antimicrob. 2014;13:16
20. Khan AA, Ponce E, Nawaz MS, Cheng CM, Khan JA, West CS. Identification and characterization of class 1 integron resistance gene cassettes among Salmonella strains isolated from imported seafood. Appl Environ Microbiol. 2009;75:1192-6.

21. Li B, Hu Y, Wang Q, Yi Y, Woo PC, Jing H, et al. Structural diversity of class 1 integrons and their associated gene cassettes in Klebsiella pneumoniae isolates from a hospital in China. PLoS One. 2013;8:e75805.

22. Wu K, Wang F, Sun J, Wang Q, Chen Q, Yu S, et al. Class 1 integron gene cassettes in multidrug-resistant gram-negative bacteria in southern China. Int J Antimicrob Agents. 2012;40:264-7.

23. Gruteke P, Goessens W, Gils J, Peerbooms P, Lemmens-Den Toom N, Van Santen-Verheuvel $M$, et al. Patterns of resistance associated with integrons, the extended-spectrum $\beta$-lactamase SHV-5 gene, and a multidrug efflux pump of Klebsiella pneumoniae causing a nosocomial outbreak. J Clin Microbiol. 2003;41:1161-6.

24. Firoozeh F, Zahraei-Salehi T, Shahcheraghi F, Karimi V, Aslani MM. Characterization of class I integrons among Salmonella enterica serovar Enteritidis isolated from human and poultry. FEMS Immunol Med Microbiol. 2011;64:237-43.

25. Firoozeh F, Shahcheraghi F, Zahraei-Salehi T, Karimi V, Aslani MM. Characterization of antimicrobial resistance profile and class I integrongs among Salmonella enterica serovars isolated from human clinical specimens in Tehran, Iran. Iran J Microbiol. 2011;3:112-7.

26. Sun J, Zhou M, Wu Q, Ni Y. Characterization of two novel gene cassettes, dfrA27 and aadA16, in a non-01, non-0139 Vibrio cholerae isolate from China. Clin Microbiol Infect. 2010:16:1125-9.

27. Adams-Sapper S, Sergeevna-Selezneva J, Tartof S, Raphael E, Diep BA, Perdreau-Remington F, et al. Globally dispersed mobile drug-resistance genes in gram-negative bacterial isolates from patients with bloodstream infections in a US urban general hospital. J Med Microbiol. 2012;61:968-74.

28. Rajaei B, Sepehri-Rad N, Badmasti F, Razavi MR, Aghasadeghi MR, Saboohi R, Rajaei T, Moshiri A, Siadat SD. Multiple-antibiotic resistance in Salmonella enterica serovars isolated in Iran harboring class 1 integrons. Br Microbiol Res J. 2015;5:186-93.

29. Firoozeh F, Zahraei-Salehi T, Shahcheraghi F. Molecular clonality and detection of class 1 integron in multidrug-resistant Salmonella enterica isolates from animal and human in Iran. Microb Drug Resist. 2014;20:517-24.

30. Shahcheraghi F, Rahmati Ghezelgeh F, Nobari S, Torabi E, Mousavi SF, Aslani MM, et al. Identification and characterization of class 1 integrons among atypical enteropathogenic Escherichia coli isolated from children under 5 years of age. Iran J Microbiol. 2014;6:156-62

31. Huang LY, Chen TL, Lu PL, Tsai CA, Cho WL, Chang FY, et al. Dissemination of multidrug-resistant, class 1 integron-carrying Acinetobacter baumannii isolates in Taiwan. Clin Microbiol Infect. 2008;14:1010-9.

32. Chang CY, Fang YT, Tsai SM, Chang LL, Yu WL. Characterization of class 1 integrons and gene cassettes in clinical isolates of Klebsiella pneumoniae from Taiwan. Diagn Microbiol Infect Dis. 2009;65:214-6.

33. Karczmarczyk M, Abbott Y, Walsh C, Leonard N, Fanning S. Characterization of multidrug-resistant Escherichia coli isolates from animals presenting at a university veterinary hospital. Appl Environ Microbiol. 2011;77:7104-12.

34. Taherikalani M, Maleki A, Sadeghifard N, Mohammadzadeh D, Soroush S, Asadollahi $P$, et al. Dissemination of class 1,2 and 3 Integrons among different multidrug resistant isolates of Acinetobacter baumannii in Tehran hospitals, Iran. Pol J Microbiol. 2011;60:169-74.

35. Memariani M, Najar Peerayeh S, Shokouhi Mostafavi SK, Zahraei-Salehi T. Detection of class 1 and 2 integrons among Enteropathogenic Escherichia coli isolates. Arch Pediatr Infect Dis. 2014;2:e16372.

36. Kang HY, Jeong YS, Oh JY, Tae SH, Choi CH, Moon DC, et al. Characterization of antimicrobial resistance and class 1 integrons found in Escherichia coli isolates from humans and animals in Korea. J Antimicrob Chemother. 2005;55:639-44.

37. Cavicchio L, Dotto G, Giacomelli M, Giovanardi D, Grilli G, Franciosini MP, et al. Class 1 and class 2 integrons in avian pathogenic Escherichia coli from poultry in Italy. Poult Sci. 2015:94:1202-8.

38. Roe MT, Vega E, Pillai SD. Antimicrobial resistance markers of class 1 and class 2 integron bearing Escherichia coli from irrigation water and sediments. Emerg Infect Dis. 2003;9:822-6.

39. Eftekhari N, Bakhshi B, Pourshafie MR, Zarbakhsh B, Rahbar M, Hajia M, et al. Genetic diversity of Shigella spp. and their integron content. Foodborne Pathog Dis. 2012;10:237-42. 